\title{
A SET ORIENTED APPROACH TO GLOBAL OPTIMAL CONTROL*
}

\author{
Oliver Junge ${ }^{1}$ And Hinke M. OsingA ${ }^{2}$
}

\begin{abstract}
We describe an algorithm for computing the value function for "all source, single destination" discrete-time nonlinear optimal control problems together with approximations of associated globally optimal control strategies. The method is based on a set oriented approach for the discretization of the problem in combination with graph-theoretic techniques. The central idea is that a discretization of phase space of the given problem leads to an (all source, single destination) shortest path problem on a finite graph. The method is illustrated by two numerical examples, namely a single pendulum on a cart and a parametrically driven inverted double pendulum.
\end{abstract}

Mathematics Subject Classification. 49J53, 49M25, 65K10, 90C39.

Received July 22, 2003. Revised October 30, 2003.

\section{IntRoduction}

The idea to solve optimal control problems by searching for shortest paths is immediately clear for specific classes of control problems. For example, consider the task of moving a particle from a given position to a desired destination, subject to a cost function that is directly related to the arclength of the path followed, or let the cost function be simply time itself so that the shortest path is defined as the fastest path. In this paper we investigate how to rephrase more general optimal control problems in terms of finding a shortest path. The main idea is to consider a discrete-time version of the model (e.g. a time- $T$-map) which is translated into a graph-theoretic description. The problem then becomes that of finding a path of minimal length from the initial state to the final state in a directed weighted graph. We show how to construct a finite graph so that standard shortest path algorithms from graph theory (like Dijkstra's algorithm [8]) can immediately be applied in order to solve the problem. The advantage of these techniques is that the approximate optimal cost and approximate optimal trajectories for all possible initial points are calculated simultaneously.

We emphasize that it is possible to use this graph-theoretic approach when the state space is $\mathbb{R}^{d}$, that is, it contains an uncountable number of points. Using a multilevel discretization of phase space one can efficiently derive finite directed weighted graphs that serve as coarse models for the evolution of the underlying control system. On each of these graphs an application of a shortest path algorithm yields an approximation to the (optimal) value function of the problem and to corresponding nearly optimal trajectories.

\footnotetext{
Keywords and phrases. Global optimal control, value function, set oriented method, shortest path.

* We would like to thank Robert Preis for providing an implementation of Dijkstra's algorithm for GAIO. We gratefully acknowledge helpful discussions with Lars Grüne, Arjan van der Schaft and Alexander Vladimirksy on the contents of this paper. H.M.O. thanks the University of Paderborn for their hospitality and financial support during her visit.

${ }^{1}$ Institute for Mathematics, University of Paderborn, 33095 Paderborn, Germany; e-mail: junge@upb.de

2 Engineering Mathematics, University of Bristol, Bristol BS8 1TR, UK; e-mail: H.M.Osinga@bristol.ac.uk
} 
Standard approaches to the solution of optimal control problems include (adaptive) finite difference schemes for the solution of the (discrete) Hamilton-Jacobi-Bellmann equation, see e.g. the work of Falcone [9] and Grüne [11]. More recently, Sethian and Vladimirsky introduced so-called ordered upwind methods that yield efficient non-iterative schemes [18].

There have been few attempts to exploit the efficiency of graph-theoretic tools in the context of optimal control. Especially in robotic navigation, the idea of finding shortest paths is used for spaces with stationary or moving obstacles. Here, the emphasis is on finding just one rather than the optimal path. Brooks and Lozano-Pérez [1] present a subdivision algorithm for finding such collision-free paths. The essence of their work lies in constructing an appropriate partition of the configuration space and finding a string of connected cells. This construction can be extremely complicated due to the geometry of the obstacles and of the moving object. The aim is to find one path, and the constructed graph, with cells as vertices and edges indicating neighboring cells, is not complete.

More recently, Tsitsiklis [21] described a graph-theoretic approach to a problem of finding an optimal trajectory; see also [16]. The moving object is viewed as a particle and any obstacles are stationary. The aim is to minimize the trajectory until it reaches the boundary of a pre-specified subset of $\mathbb{R}^{d}$, at which a terminal cost is incurred. Hence, if this boundary is one particular point, namely the desired destination, the problem is similar to what is considered in this paper. Obstacles are avoided by imposing an infinite terminal cost at the boundaries surrounding them, where it is assumed that the obstacles admit a finite description. The construction of the associated finite graph is based on a rather ad hoc discretization and the method is, unfortunately, restricted to problems where the cost function does not explicitly depend on the control.

Broucke [2] and Broucke et al. [3,4] arrive at a similar problem setting using a partitioning of the domain by bisimulation. Their method does not require that the cost function is independent of the control. Instead, they rely on the existence (and knowledge) of a particular number of first integrals that are needed to construct the bisimulation partition. Effectively, the vertices in the graph thus obtained are autonomous dynamical systems using a particular fixed constant control. It is not easy to obtain such a bisimulation partition and there is no automatic construction available. However, the (optimal) paths obtained from the graph are genuine trajectories of the underlying control system.

In this paper, we consider the problem of finding an optimal path from any initial condition to a prescribed final destination subject to an arbitrary continuous cost function which is positive everywhere except at the final destination point - in particular, the cost function may also depend on the control. We describe how to construct a finite directed weighted graph based on a very natural discretization of the problem, and use standard shortest path algorithms in order to find the solution. The construction of the graph is part of the algorithm and does not need any first integrals. The computed shortest paths yield pseudo-trajectories of the system, which might then be employed as initial guesses for standard (local) solvers in order to find true trajectories.

As mentioned, we can apply this procedure to continuous-time control systems by considering an associated time-T-map. Typically this map will be computed by applying some suitable numerical integration scheme to the given vector field. It remains to analyse to which extent the approximate value function of the resulting map actually yields a good approximation of the value function of the original continuous-time system. We prove pointwise convergence of our approach, further exploration will show on which subsets of phase space we can extend this to a statement about uniform convergence. Also, we do not yet consider the presence of obstacles, but are confident that the algorithm can be modified by removing vertices associated with the obstacles from the graph.

A more detailed outline of the paper is as follows: we start with a description of the discrete-time optimal control problem under consideration in Section 2. Section 3 contains the main algorithm together with a statement about its convergence. In Section 4 we outline the implementational aspects of our approach. Finally in Section 5 we illustrate the performance of the method by two numerical examples: a single and a double inverted pendulum. 


\section{Problem formulation}

Consider the discrete-time control system

$$
x_{k+1}=f\left(x_{k}, u_{k}\right), \quad k=0,1, \ldots,
$$

with $f: X \times U \rightarrow \mathbb{R}^{d}$ continuous. Here, $X \subset \mathbb{R}^{d}, 0 \in X$, is a particular compact region of interest, and the set $U \subset \mathbb{R}^{m}, 0 \in U$, of admissible controls is compact. We assume that the origin of the uncontrolled system is a fixed point, that is, $f(0,0)=0$. Our goal is, starting at some point $x \in X$, to impose controls $\mathbf{u}=\left(u_{0}, u_{1}, \ldots\right) \in U^{\mathbb{N}}$ such that the trajectory $\left(x_{k}(x, \mathbf{u})\right)_{k \in \mathbb{N}}$, defined as

$$
x_{0}(x, \mathbf{u})=x \quad \text { and } \quad x_{k+1}(x, \mathbf{u})=f\left(x_{k}(x, \mathbf{u}), u_{k}\right), \quad k=0,1, \ldots
$$

remains in $X$ for all $k \geq 0$ and satisfies $x_{k}(x, \mathbf{u}) \rightarrow 0$ as $k \rightarrow \infty$. If this is possible, then we say that $x$ is in the stabilizable subset $S \subset X$. Evidently, there may be $x \in X$ for which there is no control $u \in U$ such that $f(x, u) \in X$ or for which no stabilizing control sequence $\mathbf{u}$ exists, i.e. $x \notin S$. For every $x$, we let

$$
\mathcal{U}(x)=\left\{\mathbf{u} \in U^{\mathbb{N}}: x_{k}(x, \mathbf{u}) \rightarrow 0 \text { as } k \rightarrow \infty\right\}
$$

be the set of stabilizing control sequences for $x$. Hence, the set $S$ contains all $x \in X$ for which $\mathcal{U}(x)$ is nonempty.

At each step of the iteration process (1) we incur an instantaneous cost $q(x, u)$, where

$$
q: X \times U \rightarrow[0, \infty)
$$

is continuous and satisfies $q(x, u)>0$ for $x \neq 0$ and $q(0,0)=0$. In addition to stabilizing our system we aim to minimize the total cost

$$
J(x, \mathbf{u})=\sum_{k=0}^{\infty} q\left(x_{k}(x, \mathbf{u}), u_{k}\right) \in[0, \infty]
$$

that we accumulate along a trajectory.

Our goal is to compute an approximation of the (optimal) value function

$$
V(x)=\inf _{\mathbf{u} \in \mathcal{U}(\mathbf{x})} J(x, \mathbf{u})
$$

We let $\inf \emptyset=\infty$, i.e. we set $V(x)=\infty$ for $x$ with $\mathcal{U}(x)=\emptyset$, that is, for $x \notin S$. It turns out that our computed approximation is always finite for $x \in S$. As part of the computation, we obtain an approximation for the stabilizable subset $S$ and also an approximate optimizing sequence $\mathbf{u}=\mathbf{u}(x)$ for every $x \in S$.

\section{Computing the value function}

Bellman's principle of optimality (see e.g. [19]) states that for all $x$ such that $V(x)$ is finite we have the following recursive relation

$$
V(x)=\inf _{u \in U}\{q(x, u)+V(f(x, u))\}
$$

Hence, if we would know the value of $V$ in a neighbourhood of the origin, then we could extend this known domain to a larger neighbourhood using the above with $V$ as the "cost-to-go". This construction is reminiscent of algorithms for computing shortest paths in graph theory. 


\subsection{Graph-theoretic approach}

We can represent the evolution of equation (1) as a directed graph

$$
G=(X, E), \quad E=\{(x, f(x, u)) \mid x \in X, f(x, u) \in X, u \in U\},
$$

with infinitely many nodes $x \in X$ and infinitely many edges $e \in E$. The cost $q$ of the evolution is represented as a weight on the edges. Each edge $e=(x, f(x, u))$ carries the weight $w(e)=q(x, u) \geq 0$. Then, roughly speaking, for all $x$ the value function $V(x)$ is the infimum over the length of all paths connecting $x$ to 0 in the graph $G$.

In order to obtain an algorithm for the computation of $V$ we are going to approximate $G$ by a finite graph. This is the reason for restricting ourselves to a bounded subset $X \subset \mathbb{R}^{d}$ and why we can only consider controlled trajectories that remain in $X$. To this end we consider a partition $\mathcal{P}$ of $X$, that is, a finite collection of compact subsets $P_{i} \subset X, i=1, \ldots, r$, with $\cup_{i=1}^{r} P_{i}=X$, and $m\left(P_{i} \cap P_{j}\right)=0$ for $i \neq j$ (where $m$ denotes Lebesgue measure). We can now define a finite approximation to $G$, namely the graph

$$
G_{\mathcal{P}}=\left(\mathcal{P}, E_{\mathcal{P}}\right), \quad E_{\mathcal{P}}=\left\{\left(P_{i}, P_{j}\right) \in \mathcal{P} \times \mathcal{P} \mid f\left(P_{i}, U\right) \cap P_{j} \neq \emptyset\right\},
$$

where the edge $e=\left(P_{i}, P_{j}\right)$ carries the weight

$$
w(e)=\min _{x \in P_{i}, u \in U}\left\{q(x, u) \mid f(x, u) \in P_{j}\right\} .
$$

Note that, due to the compactness of $P_{i}, P_{j}$ and $U$, and the continuity of $f$ and $q$, we indeed have a minimum here. Of course, determining $E_{\mathcal{P}}$ together with the weights $w(e), e \in E_{\mathcal{P}}$, is a crucial computational step. We refer to Section 4 for a description of how these computations can be carried out in an efficient way. Let us emphasize again that $G_{\mathcal{P}}$ is a graph with finitely many nodes.

We use $G_{\mathcal{P}}$ to find an approximation of the value function $V$. For any $x \in X$ there is a subset $P(x) \in \mathcal{P}$ containing $x$. The approximation for $V(x)$ will be the length of a shortest path from the node $P(x)$ to the node $P(0) \in \mathcal{P}$ that contains the origin. The length of a path is determined as follows. Let $p=\left(e_{1}, \ldots, e_{m}\right), e_{k} \in E_{\mathcal{P}}$, be a path in $G_{\mathcal{P}}$. The length of $p$ is defined as

$$
w(p)=\sum_{k=1}^{m} w\left(e_{k}\right)
$$

We say that a path $p=p(x)=\left(e_{1}, \ldots, e_{m}\right), e_{k} \in E_{\mathcal{P}}$, in $G_{\mathcal{P}}$ connects $x$ to 0 , if $e_{1}=\left(P(x), P_{1}\right)$ and $e_{m}=$ $\left(P_{m-1}, P(0)\right)$ for some sets $P_{1}, P_{m-1} \in \mathcal{P}$. If no such path exists then $x$ is not stabilizable (in fact, none of the points in $P(x)$ is) and $V(x)=\infty$. For all $x \in X$ we approximate $V(x)$ by

$$
V_{\mathcal{P}}(x)=\min \{w(p(x)) \mid p(x) \text { connects } x \text { to } 0\},
$$

where, again, we set $\min \emptyset=\infty$. One should realize that an edge $e=\left(P_{i}, P_{j}\right)$ exists as soon as the image of the set $P_{i}$ for some control $u \in U$ intersects the set $P_{j}$. Hence, for a given path $p(x)=\left(e_{1}, \ldots, e_{m}\right), e_{i}=\left(P_{i-1}, P_{i}\right)$, there need not exist a trajectory $\left(x_{k}(x, \mathbf{u})\right)_{k \in \mathbb{N}}$ such that $x_{k}(x, \mathbf{u}) \in P_{k}$ for $k=0, \ldots, m$. This means that the value of $V_{\mathcal{P}}(x)$ may be less than that of $V(x)$, and also that we may find a path $p(x)$ connecting $x$ to 0 for some $x \in X$ that is, in fact, not stabilizable. More formally we have the following property for $V_{\mathcal{P}}$ :

Proposition 3.1. For every partition $\mathcal{P}$ of $X, V_{\mathcal{P}}(x) \leq V(x)$ for all $x \in X$.

Proof. The statement obviously holds for $x \in X$ with $V(x)=\infty$. Hence, consider $x \in X$ with $V(x)<\infty$. It suffices to show that, for arbitrary $\varepsilon>0$, there is a path $p(x)$ in $G_{\mathcal{P}}$ connecting $x$ to 0 such that $w(p(x)) \leq$ $J(x, \mathbf{u})$, where $\mathbf{u}=\left(u_{0}, u_{1}, \ldots\right) \in \mathcal{U}(x)$ is a sequence of controls such that $J(x, \mathbf{u})<V(x)+\varepsilon$. 
Let $p(x)$ be a path in $G_{\mathcal{P}}$ that is defined by following the trajectory $\left(x_{k}(x, \mathbf{u})\right)_{k \in \mathbb{N}}$, namely

$$
p(x)=\left(e_{1}, \ldots, e_{m}\right), \quad e_{k}=\left(P\left(x_{k-1}\right), P\left(x_{k}\right)\right), \quad k=1, \ldots, m,
$$

where $m$ is such that $x_{m} \in P(0)$. The length of this path is

$$
\begin{aligned}
w(p(x)) & =\sum_{k=1}^{m} w\left(e_{k}\right)=\sum_{k=1}^{m} \min _{x \in P\left(x_{k-1}\right), u \in U}\left\{q(x, u) \mid f(x, u) \in P\left(x_{k}\right)\right\} \\
& \leq \sum_{k=1}^{m} q\left(x_{k-1}, u_{k-1}\right) \leq \sum_{k=1}^{\infty} q\left(x_{k-1}, u_{k-1}\right)=J(x, \mathbf{u}) .
\end{aligned}
$$

Note that there are three factors that make $w(p(x))$ smaller than $V(x)$ : (i) the finiteness of the number of edges $m$, (ii) the computation of the weights $w\left(e_{k}\right)$ by minimizing $q$, and (iii) the fact that the shortest path $p(x)$ may actually pass through a different set of partition elements than a (near) optimal trajectory. All these are a direct result of the definition of $G_{\mathcal{P}}$ and depend on the choice of the partition $\mathcal{P}$.

\subsection{Convergence of $\boldsymbol{V}_{\mathcal{P}}$ to $\boldsymbol{V}$}

We are now going to establish a statement about the convergence of $V_{\mathcal{P}}$ to $V$ as the diameter of $\mathcal{P}$, defined as $\operatorname{diam}(\mathcal{P}):=\max _{i} \operatorname{diam}\left(P_{i}\right)$, goes to zero. To this end let $\left(\mathcal{P}^{(\ell)}\right)_{\ell \in \mathbb{N}}$ be a sequence of nested partitions of $X$ with $\operatorname{diam}\left(\mathcal{P}^{(\ell)}\right) \rightarrow 0$ as $\ell \rightarrow \infty$. By "nested" we mean that for all $\ell$ and every $P_{i}^{(\ell+1)} \in \mathcal{P}^{(\ell+1)}$ there is a $P_{i}^{(\ell)} \in \mathcal{P}^{(\ell)}$ with $P_{i}^{(\ell+1)} \subset P_{i}^{(\ell)}$. We have the following observation:

Proposition 3.2. For $x \in S$ the sequence $\left(V_{\mathcal{P}^{(\ell)}}(x)\right)_{\ell \in \mathbb{N}}$ is monotonically increasing.

Proof. Recall that $V_{\mathcal{P}^{(\ell)}}(x)=w\left(p^{(\ell)}(x)\right)$, where $p^{(\ell)}(x)$ is a path of minimal length, connecting $x$ to 0 . Suppose that for some $\ell$ there would be minimizing paths $p^{(\ell)}(x)$ in $G_{\mathcal{P}^{(\ell)}}$ and $p^{(\ell+1)}(x)$ in $G_{\mathcal{P}^{(\ell+1)}}$ such that $w\left(p^{(\ell+1)}(x)\right)<w\left(p^{(\ell)}(x)\right)$. Using $p^{(\ell+1)}(x)$ we are going to construct a path $\tilde{p}^{(\ell)}(x)$ in $G_{\mathcal{P}^{(\ell)}}$ with $w\left(\tilde{p}^{(\ell)}(x)\right)<w\left(p^{(\ell)}(x)\right)$, contradicting the minimality of $p^{(\ell)}(x)$.

Let $p^{(\ell+1)}(x)=\left(e_{1}^{(\ell+1)}, \ldots, e_{m(\ell+1)}^{(\ell+1)}\right)$, with $e_{k}^{(\ell+1)}=\left(P_{k-1}^{(\ell+1)}, P_{k}^{(\ell+1)}\right) \in E_{\mathcal{P}^{(\ell+1)}}$. Hence, $f\left(P_{k-1}^{(\ell+1)}, U\right) \cap$ $P_{k}^{(\ell+1)} \neq \emptyset$, for all $k=1, \ldots, m(\ell+1)$. Since the partitions $\mathcal{P}^{(\ell)}$ are nested, there are sets $\tilde{P}_{k}^{(\ell)} \in \mathcal{P}^{(\ell)}$ such that $P_{k}^{(\ell+1)} \subset \tilde{P}_{k}^{(\ell)}$ for $k=0, \ldots, m(\ell+1)$. This means that $f\left(\tilde{P}_{k-1}^{(\ell)}, U\right) \cap \tilde{P}_{k}^{(\ell)} \neq \emptyset$, and thus $\tilde{e}_{k}^{(\ell)}=\left(\tilde{P}_{k-1}^{(\ell)}, \tilde{P}_{k}^{(\ell)}\right)$ is an edge in $E_{\mathcal{P}}^{(\ell)}$. Therefore, $\tilde{p}^{(\ell)}(x)=\left(\tilde{e}_{1}^{(\ell)}, \ldots, \tilde{e}_{m(\ell+1)}^{(\ell)}\right)$ is a path. Furthermore, for all $k=1, \ldots, m(\ell+1)$,

$$
\begin{aligned}
w\left(\tilde{e}_{k}^{(\ell)}\right) & =\min _{x \in \tilde{P}_{k-1}^{(\ell)}, u \in U}\left\{q(x, u) \mid f(x, u) \in \tilde{P}_{k}^{(\ell)}\right\} \\
& \leq \min _{x \in P_{k-1}^{(\ell+1)}, u \in U}\left\{q(x, u) \mid f(x, u) \in P_{k}^{(\ell+1)}\right\}=w\left(e_{k}^{(\ell+1)}\right) .
\end{aligned}
$$

This yields $w\left(\tilde{p}^{(\ell)}(x)\right) \leq w\left(p^{(\ell+1)}(x)\right)<w\left(p^{(\ell)}(x)\right)$.

So far we have shown that for every $x \in S$ we have a monotonically increasing sequence $\left(V_{\mathcal{P}^{(\ell)}}(x)\right)_{\ell \in \mathbb{N}}$, which is bounded by $V(x)$ due to Proposition 3.1. The following theorem states that for points $x \in S$ the limit is indeed $V(x)$.

Theorem 3.3. For all $x$ in the stabilizable set $S$

$$
V_{\mathcal{P}^{(\ell)}}(x) \rightarrow V(x) \quad \text { as } \ell \rightarrow \infty .
$$


Proof. Suppose that for some $x \in S$ the statement does not hold, that is,

$$
\lim _{\ell \rightarrow \infty} V_{\mathcal{P}^{(\ell)}}(x)=\bar{V}(x)<V(x)
$$

We are going to construct a trajectory $\left(x_{k}(x, \mathbf{u})\right)_{k \in \mathbb{N}}$ with cost $J(x, \mathbf{u}) \leq \bar{V}(x)$, violating the definition of $V$ and thus proving our assumption to be wrong.

In order to do so, we need to work with paths $p^{(\ell)}(x)=\left(e_{0}^{(\ell)}, e_{1}^{(\ell)}, \ldots\right)$ consisting of infinitely many edges. Note that $f(0,0)=0$, so for every $\ell \in \mathbb{N}$ there is an edge $o^{(\ell)}=\left(P^{(\ell)}(0), P^{(\ell)}(0)\right)$. Furthermore, since $q(0,0)=0$, its weight is $w\left(o^{(\ell)}\right)=0$. So formally, we can extend any finite path $p^{(\ell)}(x)$ (connecting $x$ to 0 ) by appending the $\operatorname{arc} o^{(\ell)}$ infinitely many times, i.e.

$$
p^{(\ell)}(x)=\left(e_{k}^{(\ell)}\right)_{k \in \mathbb{N}}:=\left(e_{1}^{(\ell)}, \ldots, e_{m(\ell)}^{(\ell)}, o^{(\ell)}, o^{(\ell)}, \ldots\right)
$$

while the length of $p^{(\ell)}(x)$ remains the same.

Consider the sequence of minimizing paths $p^{(\ell)}(x)=\left(e_{1}^{(\ell)}, e_{2}^{(\ell)}, \ldots, e_{m(\ell)}^{(\ell)}, o^{(\ell)}, \ldots\right), e_{k}^{(\ell)}=\left(P_{k-1}^{(\ell)}, P_{k}^{(\ell)}\right)$, in $G_{\mathcal{P}^{(\ell)}}$ with $\ell \in \mathbb{N}$. Note that, by definition, $x_{0}^{(\ell)}=x_{0}=x \in P_{0}^{(\ell)}$, for all $\ell \in \mathbb{N}$. Let us start by constructing the next point $x_{1}$ of the desired trajectory $\left(x_{k}(x, \mathbf{u})\right)_{k \in \mathbb{N}}$, together with a corresponding control $u_{0}$. For every $\ell$ choose a point $\tilde{x}_{0}^{(\ell)} \in P_{0}^{(\ell)}$ and a control $u_{0}^{(\ell)}$, such that

$$
q\left(\tilde{x}_{0}^{(\ell)}, u_{0}^{(\ell)}\right)=\min _{(x, u) \in P_{0}^{(\ell)} \times U}\left\{q(x, u): f(x, u) \in P_{1}^{(\ell)}\right\}=w\left(e_{1}^{(\ell)}\right) .
$$

Set $x_{1}^{(\ell)}=f\left(\tilde{x}_{0}^{(\ell)}, u_{0}^{(\ell)}\right)$. Since $X$ is compact, the sequence $\left(x_{1}^{(\ell)}\right)_{\ell \in \mathbb{N}}$ has a convergent subsequence $\left(x_{1}^{(\ell)}\right)_{\ell \in \hat{L}_{0}}$, for some $\hat{L}_{0} \subset \mathbb{N}$. We denote its limit by $x_{1}$. We claim that there is a control $u_{0} \in \mathbb{R}^{m}$ such that $x_{1}=f\left(x_{0}, u_{0}\right)$. In fact, $\operatorname{since} \operatorname{diam}\left(\mathcal{P}^{(\ell)}\right) \rightarrow 0$ as $\ell \rightarrow \infty$, we have $\lim _{\ell \in \hat{L}_{0}} \tilde{x}_{0}^{(\ell)}=x_{0}$. Since $U$ is compact, we can choose a convergent subsequence $\left(u_{0}^{(\ell)}\right)_{\ell \in L_{0}}$ of $\left(u_{0}^{(\ell)}\right)_{\ell \in \hat{L}_{0}}$, i.e. $L_{0} \subset \hat{L}_{0}$, with limit $u_{0}$. By continuity of $f$ we must have $x_{1}=f\left(x_{0}, u_{0}\right)$.

We now continue our construction of the trajectory $\left(x_{k}(x, \mathbf{u})\right)_{k \in \mathbb{N}}$ by repeating the above argument with $x_{1}$ instead of $x_{0}$. That is, for $\ell \in L_{0}$ we choose points $\tilde{x}_{1}^{(\ell)} \in P_{1}^{(\ell)}$ and controls $u_{1}^{(\ell)}$ with $x_{2}^{(\ell)}=f\left(\tilde{x}_{1}^{(\ell)}, u_{1}^{(\ell)}\right) \in P_{2}^{(\ell)}$, that minimize $q$. We choose a convergent subsequence $\left(x_{2}^{(\ell)}\right)_{\ell \in \hat{L}_{1}}, \hat{L}_{1} \subset L_{0}$, with limit $x_{2}$ and a corresponding control $u_{1} \in U$ as the limit of a convergent subsequence $\left(u_{1}^{(\ell)}\right)_{\ell \in L_{1}}, L_{1} \subset \hat{L}_{1}$, such that $x_{2}=f\left(x_{1}, u_{1}\right)$, etc.

The construction of the desired trajectory $\left(x_{k}(x, \mathbf{u})\right)_{k \in \mathbb{N}}$ can now be completed using a standard "diagonal argument": note that we implicitly constructed a sequence $\left(L_{k}\right)_{k>0}$ of subsets of $\mathbb{N}$, such that $L_{k+1} \subset L_{k}$ for all $k \geq 0$. Choose some arbitrary element $\ell_{0} \in L_{0}$ and inductively let $\ell_{k} \in L_{k}, k=1,2, \ldots$ be the smallest number such that $\ell_{k}>\ell_{k-1}$. Define $L=\left\{\ell_{0}, \ell_{1}, \ldots\right\}$. By construction, for every $k$, the subset $\left\{\ell_{k}, \ell_{k+1}, \ldots\right\}$ of $L$ is contained in $L_{k}$. Thus, for all $k=0,1, \ldots$,

$$
\lim _{\ell \in L} \tilde{x}_{k}^{(\ell)}=\lim _{\ell \in L} x_{k}^{(\ell)}=x_{k} \quad \text { and } \quad \lim _{\ell \in L} u_{k}^{(\ell)}=u_{k}
$$

By continuity of $q$, the incremental cost of each iterate in the trajectory $\left(x_{k}(x, \mathbf{u})\right)_{k \in \mathbb{N}}$, with $\mathbf{u}=\left(u_{0}, u_{1}, \ldots\right)$, is now directly related to the limit of the weights of the associated edges in $G_{\mathcal{P}^{(\ell)}}$. Namely, for $k=0,1, \ldots$,

$$
q\left(x_{k}, u_{k}\right)=\lim _{\ell \in L} q\left(\tilde{x}_{k}^{(\ell)}, u_{k}^{(\ell)}\right)=\lim _{\ell \in L} w\left(e_{k+1}^{(\ell)}\right)
$$


We thus obtain a controlled trajectory $\left(x_{k}(x, \mathbf{u})\right)_{k \in \mathbb{N}}$ with total cost

$$
\begin{aligned}
J(x, \mathbf{u})=\sum_{k=0}^{\infty} q\left(x_{k}, u_{k}\right) & =\lim _{K \rightarrow \infty} \sum_{k=0}^{K} \lim _{\ell \in L} q\left(\tilde{x}_{k}^{(\ell)}, u_{k}^{(\ell)}\right) \\
& =\lim _{K \rightarrow \infty} \sum_{k=0}^{K} \lim _{\ell \in L} w\left(e_{k+1}^{(\ell)}\right)=\lim _{K \rightarrow \infty} \lim _{\ell \in L} \sum_{k=0}^{K} w\left(e_{k+1}^{(\ell)}\right) .
\end{aligned}
$$

Since $w\left(e_{k+1}^{(\ell)}\right)=0$ for all $k \geq m(\ell)$, we can stop the summation at $m(\ell)-1$, possibly increasing the sum when $m(\ell)-1>K$. Hence, we obtain

$$
J(x, \mathbf{u}) \leq \lim _{\ell \in L} \sum_{k=0}^{m(\ell)-1} w\left(e_{k+1}^{(\ell)}\right)=\lim _{\ell \in L} V_{\mathcal{P}^{(\ell)}}(x)=\bar{V}(x)
$$

Note that the finiteness of $J(x, \mathbf{u})$ automatically ensures that $\mathbf{u} \in \mathcal{U}(x)$. Namely, if $\mathbf{u}$ was not stabilizing, then there would be a neighborhood $N$ of the origin and a subsequence $\left(x_{k_{i}}\right)_{i}$ of $\left(x_{k}\right)_{k}$ that never enters $N$. By continuity of $q$ and since $q(x, u) \neq 0$ for $x \neq 0$, this would imply the existence of a positive number $q_{0}$ such that $q\left(x_{k_{i}}, u_{k_{i}}\right)>q_{0}>0$ for all $i$, and thus $J(x, \mathbf{u})$ would not converge. We also obtain the desired contradiction to the definition of $V$ and this completes the proof of Theorem 3.3.

\section{IMPLEMENTATION}

One central computational step is the construction of the finite graph $G_{\mathcal{P}}=\left(\mathcal{P}, E_{\mathcal{P}}\right)$, in particular determining its edges $e=\left(P_{i}, P_{j}\right) \in E_{\mathcal{P}}$ with their associated weights $w(e)$. Once this weighted graph has been computed, standard algorithms from graph theory (for example, "all source, single destination" shortest path algorithms like Dijkstra's algorithm $[5,8]$ ) can be applied in order to compute the approximate value function $V_{\mathcal{P}}$ in $(2)$. The construction of $G_{\mathcal{P}}$ breaks down into the following three steps:

(1) construction of a suitable partition $\mathcal{P}$ of the region of interest $X \in \mathbb{R}^{d}$;

(2) construction of the set $E_{\mathcal{P}}$ of edges of $G_{\mathcal{P}}$;

(3) computation of the weights $w(e)$ for the edges $e \in E_{\mathcal{P}}$.

It is the interlocking of the first two steps in a multilevel approach, as pioneered in [6] for approximating invariant sets in dynamical systems, that makes our method computationally efficient. For an in-depth description of this approach see $[6,7,20]$. It is implemented in the software package $\mathrm{GAIO}^{1}$, which has been extended for the purposes of this paper and was used for the computations in Section 5.

\subsection{Computation of the weights}

Once the graph $G_{\mathcal{P}}$ has been computed, the computation of the weights reduces to a (nonlinear) optimization problem. This can in principle be solved by standard methods (see e.g. [12]). In the examples we worked with a rough approximation only, using

$$
w(e) \approx \min _{(x, u) \in T_{i}}\left\{q(x, u) \mid f(x, u) \in P_{j}\right\}, \quad e=\left(P_{i}, P_{j}\right) \in E_{\mathcal{P}}
$$

with $T_{i} \subset P_{i} \times U$ a finite set of "test points".

Remark 4.1. If we could determine the edges $E_{\mathcal{P}}$ and associated weights exactly, Proposition 3.1 would hold. However, with the use of test points, we introduced the possibility of errors that lead to an approximation $V_{\mathcal{P}}$ of $V$ that may no longer be a lower bound.

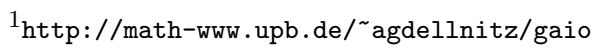




\section{EXAmples}

To demonstrate the effectiveness of our approach, we consider the problems of balancing single and double inverted pendula. We find that our method is very good to get a rough idea of the behaviour of the value function, because the computations are extremely fast. Furthermore, even with a crude partition and very few test points, the results appear to be quite accurate. A detailed error analysis of the method will be the topic of future investigations.

\subsection{Single inverted pendulum on a cart}

As a first example, we consider balancing a planar inverted pendulum on a cart that moves under an applied horizontal force $u$, constituting the control, in a direction that lies in the plane of motion of the pendulum. This problem was also studied in $[13,14]$ and we can compare the results.

The position of the pendulum is measured relative to the position of the cart as the offset angle $\varphi$ from the vertical up position. We completely ignore the dynamics of the cart and focus on the two-dimensional state space $(\varphi, \dot{\varphi}) \in \mathbb{R}^{2}$ describing the motion of the pendulum. Assuming that there is no friction, the equations of motion can be derived from first principles. Here, we use $M=8 \mathrm{~kg}$ for the mass of the cart, $m=2 \mathrm{~kg}$ for the mass of the pendulum. The center of mass lies at distance $l=0.5 \mathrm{~m}$ from the pivot. Writing $x_{1}=\varphi$ and $x_{2}=\dot{\varphi}$, the equations become

$$
\begin{aligned}
& \dot{x}_{1}=x_{2} \\
& \dot{x}_{2}=\frac{\frac{g}{l} \sin \left(x_{1}\right)-\frac{1}{2} m_{r} x_{2}^{2} \sin \left(2 x_{1}\right)-\frac{m_{r}}{m l} \cos \left(x_{1}\right) u}{\frac{4}{3}-m_{r} \cos ^{2}\left(x_{1}\right)}
\end{aligned}
$$

where $m_{r}=m /(m+M)$ is the mass ratio. We use $g=9.8 \mathrm{~m} / \mathrm{s}^{2}$ for the gravitational constant. The stabilization of this inverted pendulum is subject to the incremental cost

$$
q(x, u)=\frac{1}{2}\left(0.1 x_{1}^{2}+0.05 x_{2}^{2}+0.01 u^{2}\right)
$$

These parameters are as in [13] where the value function was computed using a completely different method.

For our computations, we need to obtain a discrete-time control system. To this end, we consider the time- $T$ map

$$
f(x, u)=\phi^{T}(x ; u)
$$

where $\phi^{t}$ is the flow associated with equation (3), $T$ is some fixed time and $u \equiv u(t)$ is the constant control action. The instantaneous cost function of the time- $T$ map is

$$
q_{T}(x, u)=\int_{0}^{T} q\left(\phi^{t}(x ; u), u\right) \mathrm{d} t
$$

where, again, $u$ denotes the constant function with value $u$ on $[0, T]$. For this example we used $T=0.1$ and each iterate was computed with the Runge-Kutta scheme of 4-th order with step size 0.02. As in [13], we choose $X=[-8,8] \times[-10,10]$ as the region of interest. We used a partition $\mathcal{P}$ of this region with boxes of size $1 / 32 \times 5 / 128$. The computation of the weights was based on a total of 4 equally spaced test points per box (i.e. the vertices) in phase space and equally spaced points in $U$ at integer multiples of 8 (including 0 ).

Let us first illustrate the effect of restricting the controls to a bounded set. Figure 1 shows two approximations of the value function $V$. In both pictures, each box $P_{i} \in \mathcal{P}$ has been given a color representing the approximate optimal cost of driving an initial condition $x \in P_{i}$ to the origin. Cost runs from 0.0 (blue) to 7.0 (red). The upper bound of 7.0 is artificial, and white regions correspond to points that either have a higher optimal cost, or are not stabilizable in $X$, so that the approximate value function is infinite here. For the left illustration of Figure 1 the controls are restricted to $U=[-64,64]$, and for the right to $U=[-128,128]$. The pictures 

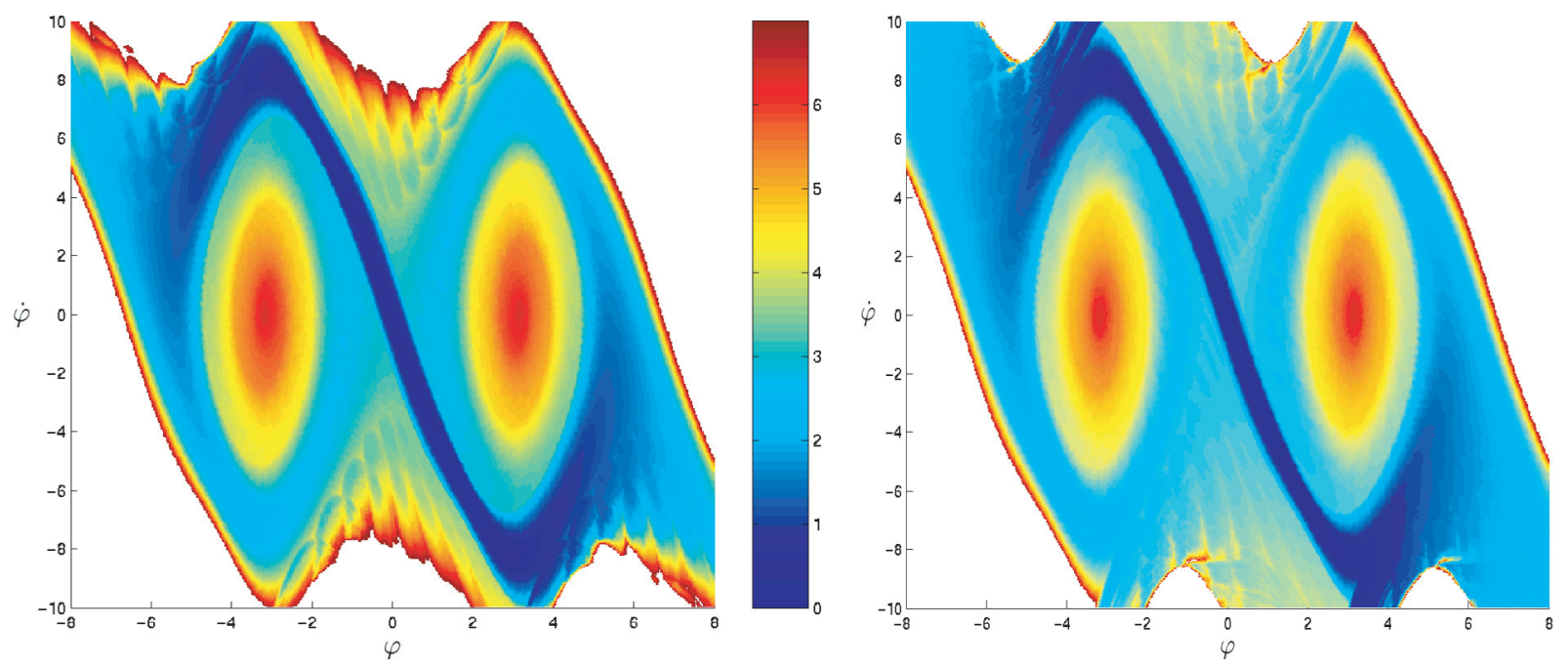

FIgURE 1. The approximate value function $V_{\mathcal{P}}$ for the single inverted pendulum on a cart. The size of the boxes in the partition $\mathcal{P}$ is $1 / 32 \times 5 / 128$. The region of interest is $X=$ $[-8,8] \times[-10,10]$ and the controls are restricted to $U=[-64,64]$ left and $U=[-128,128]$ right, respectively. The optimal cost for each initial condition is represented by a color from blue (0.0) to red (7.0).

agree quite well for relatively small (in absolute value) velocities $\dot{\varphi}$. However, for relatively large values of $|\dot{\varphi}|$, especially near $|\varphi|=0$, the optimal cost is a lot higher when the controls are restricted more severely.

The illustrations in Figure 1 also show another interesting feature. Along the boundary of the colored region, the approximate value function exhibits "oscillations". This behavior is most pronounced in the left picture. The effect that we see here is due to the restriction that an optimal trajectory must lie entirely in $X$. The bubbles with lower optimal cost are associated with optimal trajectories that are not affected by this restriction, that is, the true optimal trajectory is contained in $X$. If we enlarge $X$ this effect will again be pushed to the boundary of the region of interest.

In order to illustrate the quality of the approximation, we compared our computations with those of [13]. To this end we computed an approximation $V_{\mathcal{P}}$ of the value function $V$ using a partition $\mathcal{P}$ with boxes of size $2^{-5} \times 2^{-4}$ of the region $X=[-8,8] \times[-16,16]$, while the actual region of interest (the plotted region) remained $[-8,8] \times[-10,10]$. (It is indeed enough to only enlarge the region for $\dot{\varphi}$.) This results in a disappearance of the "oscillations" near the boundary. This time we used an integration time of $T=0.2$, realized by 10 Runge-Kutta steps. The computation of the weights was again based on a total of 4 equally spaced test points per box (the vertices) in phase space and the points $\{-120,-112,-104, \ldots, 112,120\}$ in control space. This is in accordance with the computations in [13] where the absolute value of the controls never exceeded 120. The approximation $V_{\mathcal{P}}$ is visualized in Figure 2 (left) using the same color scheme as Figure 1. Note that the choice of $T=0.2$ for the integration time leads to a result that significantly differs from the one shown in Figure 1.

The computations in [13] were done using a different method and the value function is represented as a collection of optimal cost isoclines. The computations were done up to level $V=\frac{1}{2} 3.3^{2}=5.445$. The visualization of this data is shown in Figure 2 (right). Note that the same color scheme as Figure 2 (left) was used, with a maximal cost of 7.0, so red is missing in this picture. We remark that a similar picture can be found in [13, Fig. 2], where the coloring is done proportional to the square root of the cost (in fact, the factor $\frac{1}{2}$ in the cost equation (4) is missing in this figure). It should be noted that the computations of [13] are extremely accurate, but their method is very cumbersome and the computation of the optimal cost isoclines up to $V=5.445$ took about one week. 

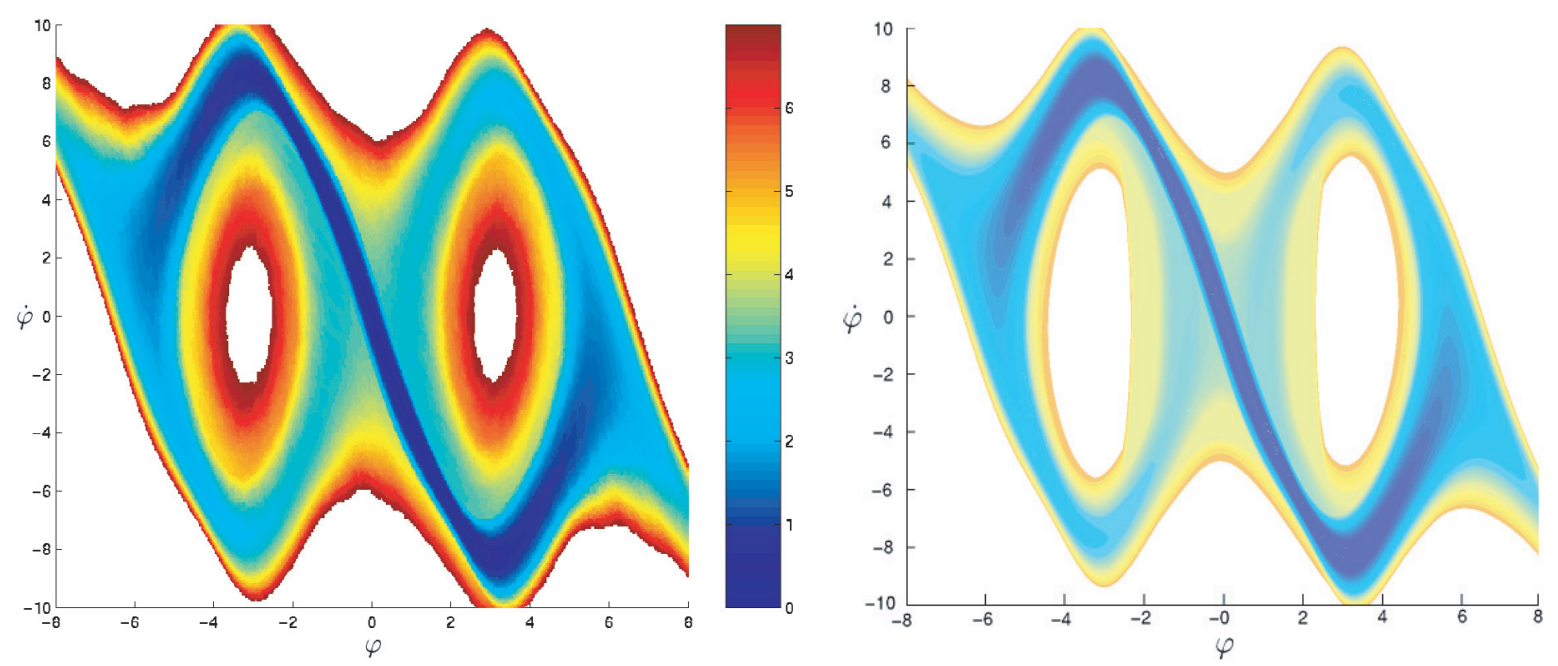

FIGURE 2. Left: approximate value function $V_{\mathcal{P}}$ for the single inverted pendulum on a cart. The size of the boxes in the partition $\mathcal{P}$ is $2^{-5} \times 2^{-4}$. Right: approximate value function as computed in [13]. Again, the optimal cost for each initial condition is represented by a color from blue (0.0) to red (7.0).

Our computation for $V_{\mathcal{P}}$ took only a couple of minutes on a recent workstation. Even when using a fairly crude partition and only few test points the comparison is already quite good. However, it seems that the "numerical" $V_{\mathcal{P}}$ is not a lower bound of $V$, as predicted by the theory. We believe that this is primarily due to the fact that we compute the weights from the test points, assigning too high an optimal cost to each edge.

\subsection{Parametrically forced inverted double pendulum}

Our method has the nice property that it is readily applicable to higher-dimensional spaces. As an example we consider the problem of balancing a parametrically driven (planar) inverted double pendulum [17]. The phase space $x=\left(\varphi_{1}, \dot{\varphi}_{1}, \varphi_{2}, \dot{\varphi}_{2}\right) \in \mathbb{R}^{4}$ is four-dimensional. The equations of motion are

$$
\left\{\begin{aligned}
2 \ddot{\varphi}_{1}+\ddot{\varphi}_{2} \cos \left(\varphi_{1}-\varphi_{2}\right) & =2\left(\frac{g+u}{\ell}\right) \sin \varphi_{1}-\dot{\varphi}_{2}^{2} \sin \left(\varphi_{1}-\varphi_{2}\right), \\
\ddot{\varphi}_{2}+\ddot{\varphi}_{1} \cos \left(\varphi_{1}-\varphi_{2}\right) & =\left(\frac{g+u}{\ell}\right) \sin \varphi_{2}+\dot{\varphi}_{1}^{2} \sin \left(\varphi_{1}-\varphi_{2}\right),
\end{aligned}\right.
$$

where $\ell=0.5$. As the corresponding discrete-time system we consider the time- $T$ map with $T=0.4$. The integration is done using the 4 -th order Runge-Kutta scheme with constant step size 0.04. The cost function is defined as in (5) with

$$
q(x, u)=\frac{1}{2}\left(0.1 \varphi_{1}^{2}+0.05 \dot{\varphi}_{1}^{2}+0.1 \varphi_{2}^{2}+0.05 \dot{\varphi}_{2}^{2}+0.01 u^{2}\right) .
$$

We use $X=[-2 \pi, 2 \pi] \times[-4 \pi, 4 \pi] \times[-2 \pi, 2 \pi] \times[-4 \pi, 4 \pi]$ as the region of interest, and $U=[-4,4]$ for the control space. The computations are done using a rather crude partition of $X$ into boxes of radii $\left(\frac{\pi}{8}, \frac{\pi}{4}, \frac{\pi}{8}, \frac{\pi}{4}\right)$. We use 81 test points (on a regular grid) in each box in phase space, and 9 equally spaced points in control space.

Unfortunately, it is not possible to visualize the value function as in the previous example, because this data set is four-dimensional. However, one can show an approximate optimal trajectory for just one arbitrarily chosen initial condition. For example, Figure 3 shows such an approximation for points $x_{0}$ near the equilibrium 


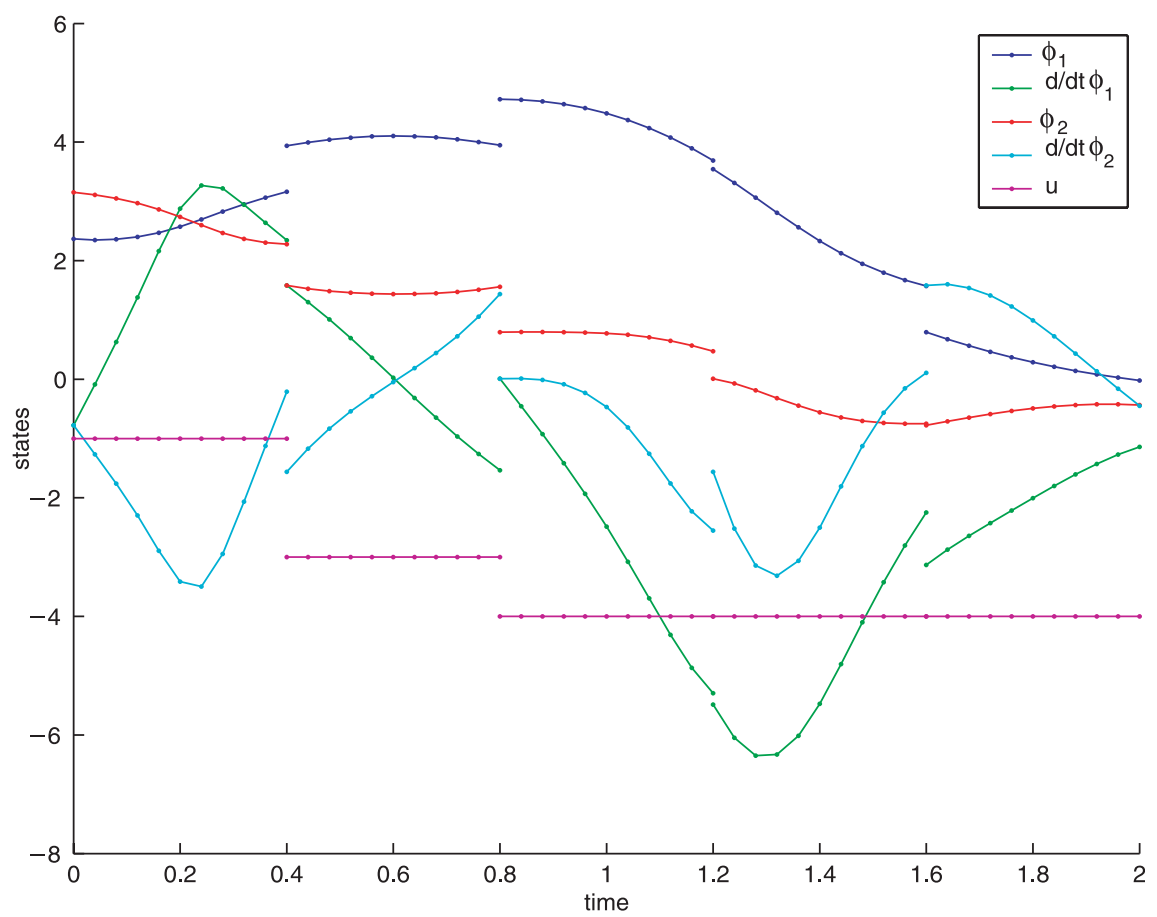

FIgURE 3. Approximate optimal pseudo-trajectory starting at $x_{e}=\left(\varphi_{1}, \dot{\varphi}_{1}, \varphi_{2}, \dot{\varphi}_{2}\right) \approx$ $(2.37,-0.78,3.15,-0.78)$, close to the vertical down position, that drives the double inverted pendulum close to the upright position.

$\left(\varphi_{1}, \dot{\varphi}_{1}, \varphi_{2}, \dot{\varphi}_{2}\right)=(\pi, 0, \pi, 0)$. The figure shows all variables $\varphi_{1}, \dot{\varphi}_{1}, \varphi_{2}, \dot{\varphi}_{2}$ plotted versus time. The approximate piecewise constant optimal control action $u$ is shown as well. For each variable we also plotted the integration steps used in the numerical integration of the time- $T$-map of (6).

In order to obtain the approximate optimal trajectory we consider the shortest path $p=p\left(x_{0}\right)$ connecting a box in the partition that contains $x_{0}$ to a box that contains the origin. By definition, each edge $e=\left(P_{i}, P_{j}\right)$ in $p\left(x_{0}\right)$ is associated with a particular state $x_{e}=\left(\varphi_{1}, \dot{\varphi}_{1}, \varphi_{2}, \dot{\varphi}_{2}\right) \in P_{i}$ and control $u_{e} \in U$ such that

$$
w(e)=\min _{x \in P_{i}, u \in U}\left\{q(x, u) \mid f(x, u) \in P_{j}\right\}=q\left(x_{e}, u_{e}\right)
$$

The approximate optimal trajectory in Figure 3 is the piecewise continuous concatenation of trajectories obtained by integrating (6) for time $T=0.4$ with initial condition $x_{e}$ and control $u \equiv u_{e}$, where $x_{e}$ and $u_{e}$ are associated with edges along the shortest path $p\left(x_{0}\right)$. For example, the first edge of the shortest path starting at a box containing $(\pi, 0, \pi, 0)$ is associated with the point $x_{e}=\left(\varphi_{1}, \dot{\varphi}_{1}, \varphi_{2}, \dot{\varphi}_{2}\right) \approx(2.37,-0.78,3.15,-0.78)$ and uses the control $u_{e}=-1$. Note that $x_{0}=(\pi, 0, \pi, 0)$ itself is not stabilizable. Hence, $V\left(x_{0}\right)=\infty$ here. Our algorithm will always assign a finite cost to the box containing $(\pi, 0, \pi, 0)$, but this value will increase more and more as the partitions are refined.

Due to the nature of our computations, the approximate optimal trajectory is only a piecewise continuous curve with discontinuities at each time- $T$ iterate. Note that such discontinuities are to be expected, because the initial condition for each iterate is determined by the weight of the edge in the optimal path, rather than by the end point of the previous iteration. As mentioned, this approximate optimal "pseudo-trajectory" should be viewed as an initial guess for standard (local) solvers for optimal control problems (see e.g. [22]). 


\section{REFERENCES}

[1] R.A. Brooks and T. Lozano-Pérez, A subdivision algorithm in configuration space for findpath with rotation. IEEE Systems, Man and Cybernetics 15 (1985) 224-233.

[2] M. Broucke, A geometric approach to bisimulation and verification of hybrid systems, in HSCC 1999, LNCS, F.W. Vaandragerand and J.H. van Schuppen Eds., Springer 1569 (1999) 61-75.

[3] M. Broucke, M.D. Di Benedetto, S. Di Gennaro and A. Sangiovanni-Vincentelli, Theory of optimal control using bisimulations, in HSCC 2000, LNCS, N. Lynch and B. Krogh Eds., Springer 1790 (2000) 89-102.

[4] M. Broucke, M.D. Di Benedetto, S. Di Gennaro and A. Sangiovanni-Vincentelli, Optimal control using bisimulations: Implementation, in HSCC 2001, LNCS, M.D. Di Benedetto and A. Sangiovanni-Vincentelli Eds., Springer 2034 (2001) 175-188.

[5] T.H. Cormen, C.E. Leierson and R.L. Rivest, Introduction to Algorithms. Cambridge, Mass. MIT Press, New York McGraw-Hill (1990).

[6] M. Dellnitz and A. Hohmann, A subdivision algorithm for the computation of unstable manifolds and global attractors. Numer. Math. 75 (1997) 293-317.

[7] M. Dellnitz, G. Froyland and O. Junge, The algorithms behind GAIO - Set oriented numerical methods for dynamical systems, in Ergodic Theory, Analysis, and Efficient Simulation of Dynamical Systems, B. Fiedler Ed., Springer (2001) 145-174.

[8] E.W. Dijkstra, A Note on Two Problems in Connection with Graphs. Numer. Math. 5 (1959) 269-271.

[9] M. Falcone, Numerical solution of Dynamic Programming equations, in Viscosity solutions and deterministic optimal control problems, M. Bardi and I. Capuzzo Dolcetta Eds., Birkhäuser (1997).

[10] Z. Galias, Interval methods for rigorous investigations of periodic orbits. Int. J. Bifur. Chaos 11 (2001) 2427-2450.

[11] L. Grüne, An Adaptive Grid Scheme for the discrete Hamilton-Jacobi-Bellman Equation. Numer. Math. 75 (1997) 319-337.

[12] P.E. Gill, W. Murray, M.A. Saunders and M.H. Wright, User's Guide for NPSOL (Version 4.0): a Fortran package for nonlinear programming, Report SOL 86-2, Systems Optimization Laboratory, Stanford University (1986).

[13] J. Hauser and H.M. Osinga, On the geometry of optimal control: the inverted pendulum example, in Proc. Amer. Control Conf., Arlington VA (2001) 1721-1726.

[14] A. Jadbabaie, J. Yu and J. Hauser, Unconstrained receding horizon control of nonlinear systems. IEEE Trans. Automat. Control 46 (2001) 776-783.

[15] O. Junge, Rigorous discretization of subdivision techniques, in Proc. Int. Conf. Differential Equations Equadiff 99, B. Fiedler, K. Gröger and J. Sprekels Eds., World Scientific 2 (2000) 916-918.

[16] L.C. Polymenakos, D.P. Bertsekas and J.N. Tsitsiklis, Implementation of efficient algorithms for globally optimal trajectories. IEEE Trans. Automat. Control 43 (1998) 278-283.

[17] K. Schiele, On the stabilization of a parametrically driven inverted double pendulum. Z. Angew. Math. Mech. 77 (1997) 143-146.

[18] J.A. Sethian and A. Vladimirsky, Ordered upwind methods for static Hamilton-Jacobi equations. Proc. Nat. Acad. Sci. USA 98 (2001) 11069-11074.

[19] E.D. Sontag, Mathematical Control Theory: Deterministic Finite Dimensional Systems, Texts in Applied Mathematics 6, Springer (1998).

[20] D. Szolnoki, Viability kernels and control sets. ESAIM: COCV 5 (2000) 175-185.

[21] J.N. Tsitsiklis, Efficient algorithms for globally optimal trajectories. IEEE Trans. Automat. Control 40 (1995) $1528-1538$.

[22] O. von Stryk, User's Guide for DIRCOL (Version 2.1): a direct collocation method for the numerical solution of optimal control problems. TU Darmstadt (2000). 